\title{
Effects of Inspiratory Muscle Training with Incentive Spirometry to Maximum Inspiratory Capacity and Quality of Life on Chronic Obstructive Pulmonary Disease Patients
}

\author{
Tresia Fransiska Ulianna Tambunan ${ }^{1}$, Moerdjajati Angka ${ }^{1}$, Anita Ratnawati ${ }^{2}, Z^{2}$ lkifi Amin ${ }^{3}$ \\ 1 Department of Physical Medicine and Rehabilitation, University of Indonesia, Cipto \\ Mangunkusumo Hospital, Jakarta, Indonesia. \\ 2 Department of Physical Medicine and Rehabilitation, Persahabatan Hospital, Jakarta, Indonesia. \\ 3 Department of Internal Medicine, Pulmonology division, University of Indonesia, Cipto \\ Mangunkusumo Hospital, Jakarta, Indonesia.
}

\begin{abstract}
Objectives: to determine whether the incentive spirometry respiratory muscle training can increase the maximum inspiratory capacity, decrease difficulty of breathing, functional lung capacity and quality of life according to St. George's Respiratory Questionnaire on Chronic Obstructive Pulmonary Disease patients.

Methods: an intervention pre-post and case control group of twenty subjects with mild COPD who came to Medical Rehabilitation Department. They divided into two groups: control group (10 subjects) and study group (10 subjects). All subjects underwent pre interventional test which are : maximum inspiratory capacity, dyspnoea rating scale, pulmonary functional test (PFT) and questionnaire for quality of life. The study group were given respiratory muscle training with incentive spirometry and breathing control exercise while the control group only given the breathing control exercise. After 8 weeks, all participant underwent post interventional test. Every subject still using the basic medication. Results: There are statistically improvement of maximum inspiratory capacity, dyspnoea rating scale and quality of life in study group compare with the control group $(\mathrm{p}<0,05)$. Mean difference of SGRQ between pre and post intervention shows significant results in activity, impact and total component $(p<0,05)$ and there is no significant results on symptoms components $(p>0,05)$. No changes were found in $\mathrm{FEV}_{1} \%$ value with the study or control group $(\mathrm{p}>0,05)$.

Conclusions: Combination between incentive spirometry respiratory muscle training and breathing control exercise can improve maximum inspiratory capacity, dyspnoea rating scale and quality of life in COPD patients within 8 weeks.
\end{abstract}

Keywords: $C O P D$, Incentive spirometry muscle training, Maximum inspiratory capacity, Quality of life

\section{INTRODUCTION}

Chronic obstructive pulmonary disease (COPD) is a respiratory disorders, characterized by progressive airway obstruction and lung hyperinflation, systemic inflammation and

Received in March 2013 and accepted for published in April 2013.

Correspondence email: Tresia Fransiska Ulianna Tambunan, fransiska_ut@yahoo.com.au increasing frequency, severity exacerbation and peripheral muscle dysfunction, all of which contribute to dyspnoea and exercise tolerance. It is largely caused by smoking and may be partially reversible. ${ }^{1}$ The airflow limitation is usually progressive and associated with an abnormal inflammatory response of the lung to noxious particles or gases. ${ }^{2}$

Lung hyperinflation increases dead space ventilation and energy consumption during hyperpnoea, leading to decreased ventilatory 
reserve and lower oxygenation in COPD patients. $^{3}$

Recent WHO figures show that an estimated 210 million people have COPD worldwide with in excess of 3 million deaths in 2005 , equal to $5 \%$ of all deaths globally that year. In the absence of interventions to cut risks, the death-toll from COPD is projected to increase by more than $30 \%$ in the next 10 years. ${ }^{4}$

Ministry of Health of Indonesia in 1995 noted that prevalence rate was $13 \%$ and mortality rate would be the fifth highest cause of death for bronchitis, bronchial asthma and pulmonary emphysema. ${ }^{5}$ In the United States, incidence and prevalence of COPD mortality in 1995 estimated 14 million patients as the fourth largest causes of death after cardiovascular, cancer and cerebral vascular disease. ${ }^{6}$

Management rehabilitation of COPD mainly to relieve symptoms, prevent deconditioning syndrome, increase functional capacity, prevent and reduce frequency of exacerbations mortality, improve ability of activity daily living, achieve optimally selfability and improve quality of life. Benefits of respiratory therapy have been reported improve and enhance the ability of cardiopulmonary system, thereby increase physical ability. ${ }^{?}$

Rehabilitation in COPD aims to improve the ability of patients, thus achieving optimal self. This objective can be achieved by the provision of training in order to reduce and control the symptoms. COPD is associated with impaired respiratory muscle function, several studies have shown exercise the respiratory muscles can be affected in patients with COPD. ${ }^{8}$ Studies on the muscles of inspiration exercises have been conducted, the American Association of Cardiovascular and Pulmonary Rehabilitation (AACVR) in 1997 stated that exercises the muscles of inspiration will enhance training capabilities, improving the degree of dyspnoea and a better quality of life. Based on this mechanism then some clinicians have made research on the benefits of breathing exercises using incentive spirometry as a non-pharmacological therapy in relation to maintaining the strength of inspiration muscle in patients with COPD. ${ }^{9}$

In general, the use of incentive spirometry is recommended for postoperative thoracotomy, but the effectiveness of its use in patients with COPD who are not operated yet known. The benefits of breathing exercises using incentive spirometry in patients with COPD is still controversial for some ahli. ${ }^{10}$ Basoglu et al, in a study proves that the use of incentive spirometry in patients with COPD apparently inspired muscle repair, the degree of dyspnoea and quality hidup. ${ }^{3}$

The incentive spirometer is a device that encourages patients with usual and other positive feedback, to maximally inflate their lungs and sustain that inflation. It is a common mode of postoperative respiratory therapy and involves deep breathing facilitated by a simple mechanical device. Maximal lung inflation is thought to open collapsed alveoli and thereby prevent and resolve atelectasis Incentive spirometry (IS) is the treatment technique which utilizes the incentive spirometry for respiratory therapy. The use of incentive spirometer is recommended for COPD patients postoperatively. However, its efficacy in COPD patients, independent of surgery, is unknown. ${ }^{11-14}$

Riera, et all ( 2001 ), examined the effect of inspiratory muscle training in patient COPD with maximum inspiration pressure loads reach $60-70 \%$ for 30 minutes/day, 6 days/ week during respectively 6 months on 30 severe CPOD shows an increase in maximal inspiratoy pressure, decrease of dyspnoea and significant improvement of functional ability and quality of life. ${ }^{10}$

Basoglu, et all ( 2005 ) observed 27 patient with clinically stable COPD, divided in two groups : IS treatment groups (incentive spirometry and medical treatment ) and control groups ( only medical treatment ) for 8 weeks. However, at the end of 2 months the $\mathrm{PaCO} 2$ had decreased, There were significant decrease $\mathrm{PaCO} 2, \mathrm{PaO} 2$ had increased and $\mathrm{PAO} 2$ had improved. Furthermore, the activity, impact and total SGRQ score were improved by the use of incentive spirometry. ${ }^{3}$

The aims of this study were to evaluate the effect of IS on maximum inspiratory capacity, difficulty of breathing (Borg's Scale), pulmonary functional test and quality of life according to St. George's Respiratory Questionnaire in 
COPD patients and then follow up to 8 weeks. Besides, patients who received both medical therapy and used an incentive spirometer were compared with patients who receives medical treatment and breathing exercise.

\section{METHODS}

All patients were over 40 years of age with mild to moderate COPD admitted from Pulmonology subdivision of Physical Medicine Rehabilitation department at RSUPN Cipto Mangunkusumo and Persahabatan. Patients with $\mathrm{VC}<10 \mathrm{ml} /$ $\mathrm{kg}$ or IC $<1 / 3$ prediction ( can't deep breathing effectively ) and cognitive impairment were excluded. The study population consisted of 20 consecutively selected patients with simple randomization who were came for rehabilitation management of COPD. Drop out criteria did not complete home programme exercise for 7 days respectively and subject didn't come for evaluation 2 weeks respectively to the institution.

A total of 10 patients were allocated to the IS treatment group. They were asked to undertake IS and were also given medication for COPD. The remaining 16 patients ( control group ) received only medication and breathing exercise.

The patients were evaluated on five occasions. The first visit was on admission to the policlinic : a full history was taken and previous medical problems, medications, smoking history and comorbid diseases were recorded. After a physical examination, a chest X-ray was obtained PFTs were performed with sealed spirometer ( Enraf Nonius BV, Model Spiro 601 ). Borg's Scale was used to assess perception of dyspneu and St. George's Respiratory Questionnaire ( SGRQ ) was used in the evaluation quality of life. All baseline measurement of maximum inspiratory capacity, pulmonary function, dyspnoea, and SGRQ were performed immediately before treatment. The next three visits were performed to the institution every 2 weeks and the compliance of the patients with IS and breathing exercise treatment was questioned and two times a week were performed via telephone calls. Clinical evaluation, maximum inspiratory capacity, PFT, Borg's Scale and SGRQ were all repeated at the polyclinic on the third and fifth visit, which was on week 4 and week 8 .

The effects of IS on maximum inspiratory capacity, pulmonary function, perception of dypsnoea and quality of life were assessed by comparing pre-and post treatment results for the IS treatment group and the control group. The local ethics committee approved the study and all subjects gave informed consent.

\section{Incentive Spirometry}

The patients performed IS using volumetric incentive spirometry ( DHD 222500H, USA), which was available in $2500 \mathrm{ml}$ (Fig.1). ${ }^{15}$ They were initially instructed on the use IS by research doctors. After a quiet expiration, they were encouraged to take slow maximal inspirations through the mouthpiece of the device and to hold each breath as long as possible for at least five seconds ). Allow the piston to fall to the bottom of the column. The patients were encouraged to use the device for 5 times/ day with 10 breath per session. ${ }^{16}$ The device of IS started on the first day patient come to the policlinic and was continued at home for 8 weeks. The compliance of the patients was assessed by telephone contact every week. One of the family members was also asked to checked that IS was being used. ${ }^{17-19}$

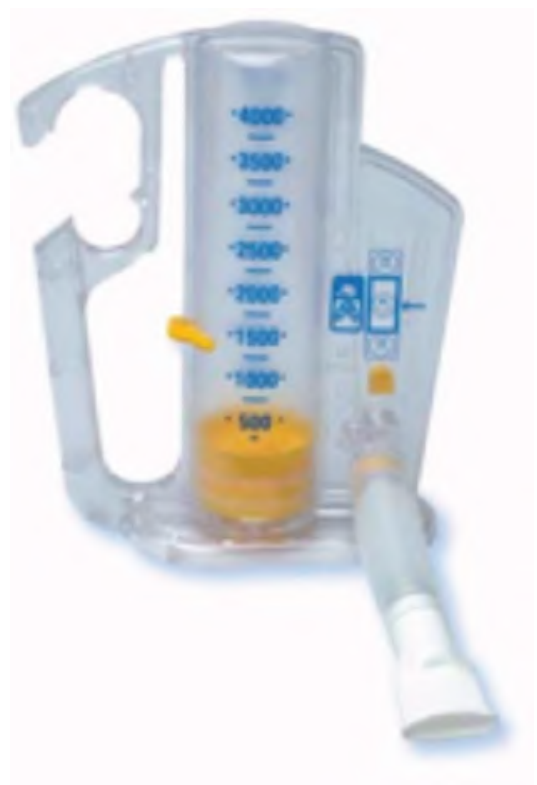

Figure 1. Incentive Spirometry 


\section{Borg's Scale}

The perception of dyspnoea was evaluated using a Borg's Scale measured perceived exertion. The original scale introduced by Gunnar Borg rated exertion on a scale 6-20. This is especially used in clinical diagnosis of breathlessness and dyspnea. ${ }^{20}$

The Borg Rating of Perceived Exertion ( RPE ) is a way of measuring physical activity intensity level. Perceived exertion is how hard you feel like your body is working. It is based on the physical sensations a person experiences during physical activity, including increased heart rate, increased respiration or breathing rate, increasing sweating, and muscle fatigue. $^{21,22}$ This scale is used to evaluate changes in the degree of tightness in chronic obstructive pulmonary disease. This scale is a valid indicator to determine the level of physical exertion or the use of force. This scale can be used effectively because of its reliability is high. Scale value range is between 0 and 10 , subjects were asked to express symptoms of shortness perceived by selecting numbers in a stretch of $0-10 .{ }^{23}$

\section{St George's Respiratory Questionnaire}

Disease-specific instrument designed to measure impact on overall health, daily life, and perceived well-being. Developed for use by patients with fixed and reversible airway. It has three components : symptoms, activity and impact. The response to the items can be aggregated into an overall score and there sub scores for symptoms, activity and impact in the range $0-100 \%$. Higher scores indicate a poorer quality of life. ${ }^{24-27}$

\section{RESULTS}

Although 25 patients with COPD was recruited for the study, 2 patients did not return after taking an early, one reason the dyspnoea increasingly become heavy and the other because of the distance from the institution. 3 people were excluded from the study of each one person in the case group and 2 in the control group performed after homogeneity test, so the number of subjects who completed the study program as many as 20 people, 10 people each for cases and controls. The remaining 20 patients ( mean age $60,27 \pm 5,55$ years) completed the study. The characteristics of the patients are shown in Table 1 . A total 17 patients $(85 \%)$ was quite active in the daily activities that tend to have a higher CMI rather than inactive.

However, they were all clinically stable with moderate COPD and under medical treatment for these concomitant disease. There were no significant differences in terms of CMI, BORG and SGRQ parameters pretreatment between two groups with paired t-test, this is done to homogenize the distribution of the study subjects $(p>0.05)$. There were no significant for PFT parameters measurement between the pre- and post- treatments of the two groups $(\mathrm{p}>0,05)$. Dyspnoea (assessed by Borg's Scale), maximum inspiratory capacity, total and component SGRQ scores demonstrated significant differences between two groups after this study. There were no significant difference for SGRQ symptoms score for the control group at the end of 8 weeks with non parametric wilcoxcon test. When all the pretreatment measurement for the IS group were compared to the post treatment measurement, there was no significant differences only for PFT parameters. However, the Borg's Scale, maximum inspiratory capacity (CMI), the symptoms, activity, impact and total SGRQ scores were improved by used on incentive spirometry $(\mathrm{p}<0.05)$. In contrast, for the control group, PFT and SGRQ symptoms scores showed no significant difference between the pre-and posttreatment assessment (Table 2). 
Table 1. Subjects' Characteristics in the Incentive Spirometry Treatment Group and the Control Group

\begin{tabular}{lcc}
\hline \multicolumn{1}{c}{ Paramaters } & IS Group (\%) & Control Group (\%) \\
$\mathbf{n}=\mathbf{1 0}$
\end{tabular}

Table 2. Comparison of Pre and Post Treatment in The Incentive Spirometry Treatment Group and The Control Group

\begin{tabular}{|c|c|c|c|c|}
\hline \multirow{2}{*}{ Parameters $^{\dagger}$} & \multicolumn{2}{|c|}{ IS Group ( $n=10$ ) } & \multicolumn{2}{|c|}{ Control Group $(\mathbf{n}=10)$} \\
\hline & Pre treatment & Post treatment & Pretreatment & Post treatment \\
\hline Borg's Scaleł & $3.2 \pm 1.22$ & $1.1 \pm 0.66$ & $3.0 \pm 0.94$ & $2.5 \pm 0.85$ \\
\hline CMI & $605 \pm 180.2$ & $1480 \pm 378$ & $465 \pm 158.2$ & $630 \pm 131.65$ \\
\hline SGRQ & & & & \\
\hline Symptoms & $394.72 \pm 154.93$ & $224.58 \pm 113.66$ & $581.28 \pm 917.85$ & $293.73 \pm 117.41$ \\
\hline Activity & $769.04 \pm 267.88$ & $399.85 \pm 220.47$ & $850.88 \pm 152.43$ & $794.15 \pm 146.59$ \\
\hline Impact & $940.36 \pm 502.94$ & $364.11 \pm 218.72$ & $966.5 \pm 444.52$ & $844.19 \pm 387.05$ \\
\hline Total & $2104.12 \pm 837.21$ & $988.54 \pm 497.74$ & $2114.81 \pm 696.88$ & $1894.34 \pm 603.82$ \\
\hline FEV1\% & $73.7 \pm 6.43$ & $72.9 \pm 6.31$ & $69.1 \pm 8.15$ & $68.9 \pm 7.78$ \\
\hline
\end{tabular}

$\dagger$ Values are expressed as mean $\pm \mathrm{SD}$

* Higher SGRQ scores indicate a poorer quality of life

* $\mathrm{p}<0.05{ }^{* *} \mathrm{p}>0.05$

IS, incentive spirometry ; CMI, capacity maximum inspiration; SGRQ, St. George's Respiratory Questionnaire

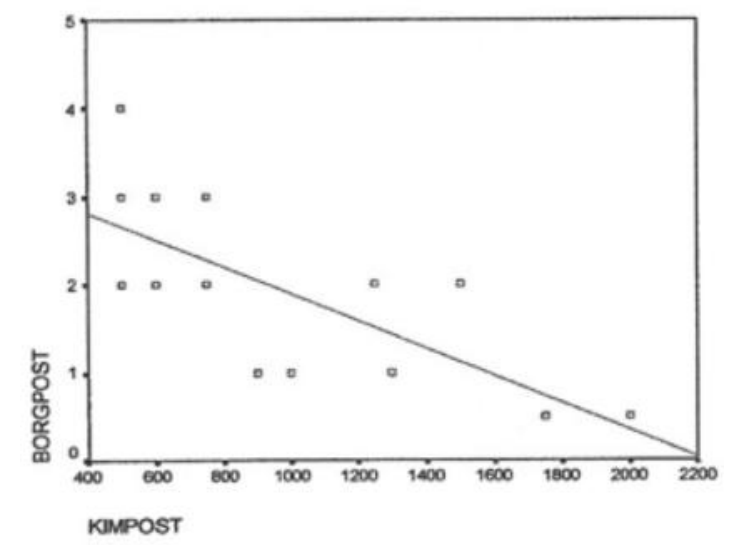

Figure 2. Correlation Between CMI and Borg's Scale Post-treatment 


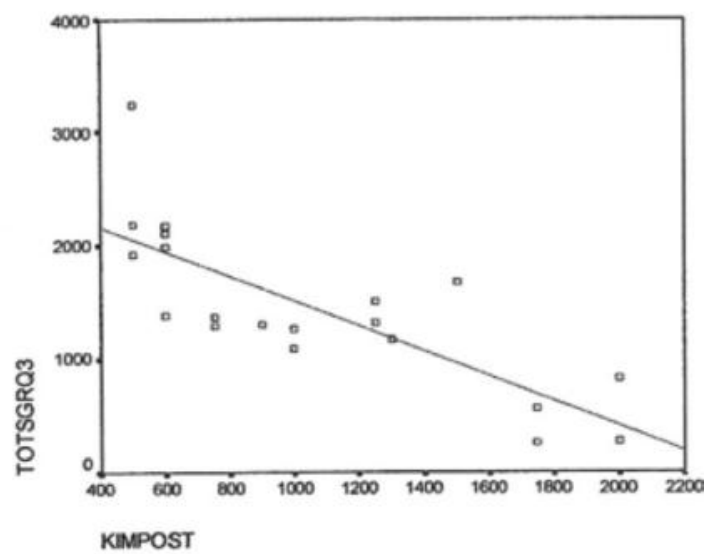

Figure 3. Correlation Between CMI and Total SGRQ Post-treatment

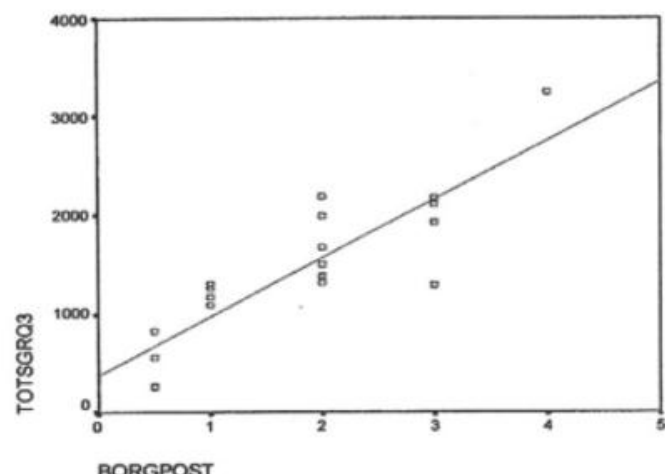

Figure 4. Correlation Between Borg's Scale and Total SGRQ Post-treatment

\section{DISCUSSION}

COPD is associated with exacerbations. Some patients are prone to frequent exacerbations and these individuals have impaired lung function, hyperinflation and increased energy consumption that lead to reduced ventilator capacity and are associated with shortness of breath and worse quality of life, greater limitation of their daily activity and faster disease progression that patients with less frequent exacerbations. ${ }^{28}$

Pulmonary rehabilitation improves health status and exercise tolerance, but no respiratory function in patient with COP and it is one of the most effective interventions in moderate COPD. ${ }^{29}$ In this study, the effects of IS in moderate COPD for 8 weeks together with medical treatment improved maximum inspiratory capacity, dyspnoea and health-related quality of life, although pulmonary function parameters remained unchanged. There were significant differences for dyspnoea for two groups, although in the IS group the dyspnoea decreased rather more than the control group, it's because the IS group were given two types of exercises : breathing control exercise and inspiratory training exercises using volumetric IS. SGRQ symptom scores there were no significant differences in the control group after non parametric Wilcoxon test statistic, this is because the distribution is not normal, while in the case of significant decline, it is according to research conducted by Jones et al, where the value for the SGRQ activity component, and the total impact each has a strong correlation of symptoms while the component has a weak correlation values. ${ }^{25}$

There were negative and significant 
correlation $(r=-0.770 ; p<0.05)$ between MIC to Borg's Scale with statistical test, which are still active COPD patients tend to have a higher KIM and decrease the degree of dyspnoea lower than the inactive (Fig.2). In a statistical significant difference, there is a significant negative correlation between KIM and the value of total SGRQ $(\mathrm{r}=-0793, \mathrm{p}<0.05)$ (Fig.3) and a positive correlation ( $\mathrm{r}=0867$, $\mathrm{p}$ $<0.05$ ) between the total value of SGRQ to the Borg's scale (Fig.4), where the value of higher KIM and low Borg's scale obtained value of total SGRQ lower.

The effects of IS as an inspiratory muscle training device was evaluated in the present study. IS is designed to mimic natural sighing or yawning by encouraging the patient to take long, slow, deep breaths and it can be used for inspiratory muscle training. Incentive spirometry is also called the Sustained Maximal Inspiration. The use of IS increases transpulmonary pressure, inspiratory volumes and inspiratory muscle performance..$^{17,30}$

The tool is easy to be taught and used by people without supervised, other than that the tool is easy to carry, so patient can do the exercises as often as possible in anywhere. Due to the use of this tool can assist in measuring and monitoring the volume of inspiration, it can also be objectively measure the ability to inspiratory maximal inspiration that can be achieved by the patient. ${ }^{17,18}$

In healthy subjects, there was a $50 \%$ improvement in inpiratory maximum pressure that can be obtained after 4 weeks of exercise every day. Strengthening exercises is usually followed by improvement endurance of patients. ${ }^{31}$

Scherer et al, studied the effects of respiratory muscle training using portable device for 8 weeks in COPD patients, while the control group performed breathing exercise using IS. They showed that breathing exercises improved the maximum inspiratory pressure (PImax) and dyspnoea in the incentive spirometry group as a result of an improvement in their inspiratory muscle performance. Although in can be argued that these changes were due to a placebo effect or based on motivation, the fact that PImax or respiratory muscle endurance, improved suggests that a mild training effect occurred. ${ }^{32}$
Tiwary et al, reported a remarkable improvement in subjective feeling of well-being and breathlessness with the use of incentive spirometry in COPD patients. These subjective finding reflect an improvement in quality of life as reported in this study. ${ }^{33}$

Igarashi et al, assessed the effects of IS on pulmonary function and analyses blood gas in healthy adults of advanced age in COPD patients. Both the control subjects and COPD patient showed a significant decreased in alveolar arterial oxygen gradient and increases in pulmonary function parameters and $\mathrm{PaO} 2$ values. ${ }^{34}$

The goals of pulmonary rehabilitation programmes are mainly to improve quality of life of the patients, rather than functional parameters. It has been shown that pulmonary rehabilitation, with additional inspiratoy muscle training can be more improve quality of life in patients with COPD. An improvement in inspiratory muscle strength and endurance might reduce symptoms and improve functional capacity in patients with moderate COPD, even if airway obstruction does not improve. Inspiratory muscle training is recommended for COPD patients. ${ }^{31}$ The combination of breathing control exercises combined with breathing exercises using volumetric IS for 8 weeks more significantly better in improving the ability of the maximum inspiration, decreasing the degree of breathlessness and quality of life but does not improve the capacity of pulmonary function in patients with moderate COPD. There were some limitations to this study. The number of subjects studied was small, all of the subjects with moderate COPD and the costs are relatively expensive and invasive examination for blood gas analysis, this is one of the limitations in this study so as to conduct more in-depth studies can not be done. But with the existing results in this study certainly expected to add an alternative form of exercise at home for respiratory rehabilitation program in dealing with patients with COPD. Prospective further studied are needed to compare the response of patients with COPD severity of the breathing exercises with IS and to see the continuity of these breathing exercises to maintain maximal inspiration capabilities and long-term quality of life of patients with COPD. 


\section{CONCLUSIONS}

An incentive spirometry is a simple device that can easily used for inspiratory muscle training. It was concluded that combination of IS and breathing control improved inspiratory muscle performance in maximum inspiratory capacity, dyspnoea and lead to have better quality of life in patients with moderate COPD without altering pulmonary function parameters.

\section{REFERENCES}

1. Ferreira IM. Up date nutritional support for patients with COPD. COPD respiratory medicine update 2008; vol4(no4):127.

2. Napitupulu L, Wahyudati S. Effects of Ergo-Cycle exercise on lung function and physical fitness of chronic obstructive pulmonary disease. Indonesian Journal Of Physical Medicine \& Rehabilitation 2012; vol1(no1):101-9.

3. Basoglu OK, Atasever A, Bacakoglu F. The efficacy of incentive spirometry in patients with COPD. Respirology 2005;10:349-53.

4. WHO Fact sheet No. 315, May 2008, ( http ://www.who.int/mediacentre/factsheets/ fs315 )

5. Survei Kesehatan Rumah Tanga Departemen Kesehatan RI 1995.

6. Millicent H. Epidemiology of obstructive pulmonary disease. Principles \& Practice of pulmonary rehabilitation. WB Saunders Company, Philadelphia 1993:10-17.

7. Casabury R. Skeletal muscle function in COPD. Chest 2000:267-70.

8. Sassi-Dambron. Treatment of dyspnoea in COPD. A Controlled Clinical Trial of Dyspnoea Management Strategies. Chest 1995;107:724-29.

9. NHLBI Workshop Summary. Respiratory muscle fatigue. Am Rev Respiratory Disease 1990 ; 142:474-80.

10. Riera HS, Rubio TM, Ruiz FO, Ramos PC, Otero DC, Hernandez TE, Gomez JC. Effect of dyspnoea, exercise performance and quality of life. In inspiratory muscle training in patients with COPD. Chest 2001;120:748-56.
11. Overend TJ, Anderson CM, Lucy SD, Bhatia C, Jonsson BI, Timmermans C. The effect on incentive spirometry on postoperative pulmonary complications : a systematic review. Chest 2001;120:971-8.

12. Chutter TAM, Weissman C, Starker PM, Gump FE. Effect of incentive spirometry on diaphragmatic function after surgery. Surgery 1989:105:488-93.

13. Hall JC, Tarala R, Harris J, Tapper J, Christiansen K. Incentive spirometry versus routine chest physiotherapy for prevention of pulmonary complications after abdominal surgery. Lancet 1991; 337:953-6.

14. Weindler J, Kiefer RT. The efficacy of postoperative incentive spirometry is influenced by the device-specific imposed work of breathing. Chest 2001;119:185864.

15. Incentive spirometry available from www. shuttertocks.com

16. How to use incentive spirometry available from www.cleavelandclinic.org.health.

17. AARC Clinical Practice Guidelines. Incentive Spirometry. Respiratory Care 1991;36:402-05.

18. Selection and use of incentive spirometry equipment. Available from : http:// selection $\% 20$ and 20 use $\% 20$ of $\% 20$ incentive $\% 20$ spirometry $\% 20$ equipment.

19. Hyperinflation therapy. Incentive spirometry. Available from : http://faculty. icc.edu/gca.

20. Borg's Scale available from www. en.wikipedia.org/wiki/borg_scale

21. Perceived Exertion (Borg Rating Of Perceuved Exertion Scale) 2011 March Available from www.cdc.gov/ physicalactivity/everyone/measuring/ exertion $/ \mathrm{html}$

22. BORG.G. Psychophysical bases of perceived exertion. Medicine and science in sports and exercise.1982;14(5):377-81.

23. Donner CF, Carone M, Zotti AM. Methods of assessment of quality of life. Europe Respiratory Rev 1997;7:42-5.

24. Andresen EM et al. Health related quality of life outcome measure. Archive Physical Medicine Rehabilitation 
2008;81(Supp12):S30-45.

25. Jones PW et al. A self-complete measure health status for chronic airflow limitation. The St George's Respiratory Questionnaire Respiratory Disease 1992:1321-7.

26. Spencer L, McKeough Z, Alison J. Priority heath care program for COPD pulmonary rehabilitation component. Respirology 2003;8:A40.

27. Heron N, Roberts $M$, Jhonston $C$, et al. Under utilization of health care services in patients with severe COPD referred to pulmonary rehabilitation. Respirology 2003;8:A42.

28. Dahlen I, Janson C. Anxiety and depression are related to the outcome of emergency treatment in patient with obstructive pulmonary disease. Chest 2002;122(5):1633-7.

29. Di Meo F, Pedone C, Lubich S, Pizzoli C, Traballesi M, Incalzi RA. Age does not hamper the response to pulmonary rehabilitation of COPD patients. Age ageing 2008 Jun 19.
30. Bastian R, Jacques J. Incentive spirometry performance. A reliable indicator of pulmonary function in the early postoperative period after lobectomy. Chest March 1997:559-63.

31. Aldrich TK. Inspiratory muscle training in chronic obstructive pulmonary disease. In: Bach JR editor. Pulmonary Rehabilitation : the obstructive and paralytic conditions. Philadelphia: Hanley \& Belfus Inc; 1996.p.123-32.

32. Scherer TA, Spengler CM, Owassapian $\mathrm{D}$, et al. Respiratory muscle endurance training in COPD disease. American Journal Respiratory Critical Care Medicine 2000;162:1709-14.

33. Tiwary RS, Lakhera SC, Kain \&Sinha TC. Effect of incentive breathing of lung functions in COPD disease. Journal Association Physicians India 1989;37:68971.

34. Igarashi T, Konishi A, Suwa K. The effects on incentive spirometry on pulmonary functions. Masui 1994;43:770-3. 